\title{
Predominance of M2-polarized macrophages in bladder cancer affects angiogenesis, tumor grade and invasiveness
}

\author{
HISASHI TAKEUCHI $^{1}$, MICHIO TANAKA ${ }^{2}$, AYAKO TANAKA ${ }^{1}$, AKISA TSUNEMI ${ }^{2}$ and HIDENOBU YAMAMOTO ${ }^{1}$ \\ Departments of ${ }^{1}$ Urology and ${ }^{2}$ Pathology, Tokyo Metropolitan Hiroo Hospital, Tokyo 150-0013, Japan
}

Received January 9, 2015; Accepted January 19, 2016

DOI: $10.3892 / \mathrm{ol} .2016 .4392$

\begin{abstract}
Tumor-associated macrophages (TAMs) often assume an immunoregulatory M2 phenotype. Thus, the aim of the present study was to clarify the correlation of vascularity and TAMs, in particular the M2 phenotype in the stroma and tumor areas, with the clinical and pathological outcomes of patients with bladder cancer. The TAM counts and microvessel counts (MVCs) were determined immunohistochemically in 21 patients with bladder cancer. The number of infiltrating TAMs was measured using immunohistochemistry with anti-cluster of differentiation (CD)68 and anti-CD163 antibodies, to identify a macrophage lineage marker and an M2-polarized-specific cell surface receptor, respectively. $\mathrm{CD}^{+} 8^{+}$and $\mathrm{CD} 63^{+}$macrophages were evaluated in the stroma and tumor areas, and areas with a high density of infiltrating cell spots were counted. MVCs were determined using immunohistochemistry with anti-CD34 antibodies. The results revealed that the higher ratio of $\mathrm{CD} 163^{+} / \mathrm{CD} 68^{+}$macrophages in the stroma, tumor and total tumor tissues were correlated with a higher stage and grade $(\mathrm{P}<0.05)$. In addition, the low ratio of $\mathrm{CD}^{2} 8^{+} / \mathrm{CD} 34^{+}$microvessels was correlated with a higher stage $(\mathrm{P}<0.05)$. There was also a positive correlation between TAMs and MVC $\left(r^{2}=0.25 ; \mathrm{P}<0.05\right)$. These results suggest that the TAM polarized M2 phenotype affects microvessels, pathological outcome, tumor grade and invasiveness.
\end{abstract}

\section{Introduction}

Bladder cancer is the fourth most common malignancy in males and the ninth most common in females in the United States. In total, 74,690 cases were diagnosed in 2014 (1). Although the cancer can be treated effectively using transurethral resection (TUR), patients are at risk of recurrence or progression to muscle-invasive disease or metastasis $(2,3)$.

Correspondence to: Dr Hisashi Takeuchi, Department of Urology, Tokyo Metropolitan Hiroo Hospital, 2-34-10 Ebisu, Shibuya-ku, Tokyo 150-0013, Japan

E-mail: t-hisa@tokyo-med.ac.jp

Key words: angiogenesis, microenvironment, bladder cancer, tumor-associated macrophages, M2-polarized macrophages
Tumor biology and progression, and the therapeutic response are affected by the tumor microenvironment $(4,5)$, including stromal cells, infiltrating leukocytes and blood vessels, which all contribute to the tumor stroma (5). Tumor-associated macrophages (TAMs) are one of the main components of this tumor stroma, and contribute to the progression of a number of cancer types $(5,6)$. The induction of angiogenesis was initially hypothesized to be via tumor cells themselves. However, certain stromal components are likely to be involved in regulating the behavior of tumors also $(7,8)$. For example, macrophages play a significant role in tumor angiogenesis and inflammatory reactions $(9,10)$. Taken together, these observations suggest that TAMs are important for tumor angiogenesis and invasion, and that they affect the prognosis.

Macrophages can alter their phenotype depending on environmental signals (11), and can be divided into classically-activated macrophages (M1) and alternatively-activated macrophages (M2) (12). M1-macrophages are pro-inflammatory and tumoricidal, whereas M2-macrophages release anti-inflammatory molecules, regulate tissue remodeling and reduce inflammation (13). In the tumor microenvironment, the majority of TAMs exhibit an M2-like phenotype (6), exert anti-inflammatory and protumor effects, and facilitate tumor growth, angiogenesis, immunosuppression and matrix remodeling (14). As such, a detailed assessment of macrophage phenotypes in the tumor, stroma and microenvironment is required in order to fully comprehend the manner in which M2 macrophages affect tumor behavior. Nevertheless, previous reports have been based solely on the expression of cluster of differentiation (CD)68, a macrophage lineage marker that does not discriminate between the M1 and M2 phenotypes, thereby generating bias with regard to resultant observations $(15,16)$.

The current study evaluated the overall TAM population (CD68; M1 and M2 macrophages) and the M2 phenotype (based on CD163 expression) in the stroma and tumor tissue of bladder cancer patients. The objective of the study was to determine the association among TAMs (including CD163 expression), microvessel counts (MVCs), pathological outcome, tumor grade and invasiveness.

\section{Materials and methods}

Tissue samples. Surgical specimens were obtained from 21 patients (19 males and 2 females; mean age, 74.0 years; range, 50-89 years) with bladder cancer, including 
17 non-muscle invasive cancers (Ta and T1) and 4 invasive bladder cancers (T2), who underwent transurethral resection at the Department of Urology, Tokyo Metropolitan Hiroo Hospital (Tokyo, Japan). Written informed consent was obtained from all the patients and all procedures used in the present study were approved by the Ethical Committee of Tokyo Metropolitan Hiroo Hospital. All tumors were transitional cell carcinomas. None of the patients had received chemotherapy, radiotherapy or any medication that may have otherwise affected the macrophage count prior to surgery. The profiles of the patients from whom tumor samples were obtained are summarized in Table I. Solitary tumors were present in 12 individuals and multiple tumors in 9 subjects. Primary and recurrent tumors were exhibited in 12 and 9 individuals, respectively. The cancer stage was determined on the basis of the tumor-node-metastasis classification system [International Union Against Cancer (17)]: 11 cases were Ta, 6 were T1 and 4 were T2. The tumor was grade I in 2 patients, grade II in 11 subjects and grade III in 8 individuals. A pathologist evaluated the tissue samples pathologically (stage and grade). Tumor recurrence was examined using computed tomography scans, cystoscopy, urinary cytology and intravenous pyelography. The mean follow-up period in the present study was 6.5 months (range, 1-42 months).

TAM count. Sections of the surgical specimens were stained with hematoxylin and eosin for histomorphological evaluation. In the bladder cancer samples, TAM and M2 macrophages were assessed using monoclonal mouse anti-human CD68 (catalog no., M0876; clone, PG-M1; Dako, Glostrup, Denmark) and monoclonal mouse anti-human CD163 (catalog no., CD163-L-CE; clone, 10D6; Novocastra ${ }^{\mathrm{TM}}$; Leica Biosystems, Nusslock, Germany) antibodies at a dilution of 1:100 in phosphate-buffered saline. Tissue sections were deparaffinized in xylene and then rehydrated using graded alcohol solutions. Endogenous peroxidase activity was blocked by immersing the slides in $3 \% \mathrm{H}_{2} \mathrm{O}_{2}$ for $10 \mathrm{~min}$. Next, the tissues were incubated in $0.125 \%$ trypsin at $37^{\circ} \mathrm{C}$ for $20 \mathrm{~min}$, followed by incubation with the primary CD68 and CD163 antibodies for $60 \mathrm{~min}$ at room temperature. The tissues were then incubated with horseradish peroxidase-conjugated secondary antibodies (Histofine ${ }^{\circledR}$ Simple Stain Mouse MAX PO; Nichirei Biosciences Inc., Tokyo, Japan) for $30 \mathrm{~min}$ at room temperature. After color development with diaminobenzidine, the slides were counterstained with hematoxylin and mounted using aqueous mounting media. The immunoglobulin fraction of normal mouse serum was used as a negative control. For TAM counts, three areas with the highest density of macrophages were analyzed at low magnification; macrophages were then counted at x200 magnification (20X objective and 10X ocular pieces) (Fig. 1A).

MVCs. Tumor-associated angiogenesis was determined using MVCs according to the method described by Weidner et al, with slight modifications (18). Anti-CD34 antibodies (monoclonal mouse anti-human CD34; clone QB end 10; Dako) were specifically used rather than anti-factor VIII antibodies to detect endothelial cells in the bladder tumors. Vessels were counted in three areas of maximal neovascularization (hot spots) in which the highest number of discrete microvessels
Table I. Clinical and pathological features of the 21 patients with bladder cancer.

\begin{tabular}{lc}
\hline Characteristics & Value \\
\hline Median age (range), years & $74(50-89)$ \\
Gender (male:female), $\mathrm{n}$ & $19: 2$ \\
Pathological T stage, $\mathrm{n}(\%)$ & \\
pTa & $11(52.4)$ \\
pT1 & $6(28.6)$ \\
pT2 & $4(19.0)$ \\
Tumor grade, $\mathrm{n}(\%)$ & \\
I & $2(9.5)$ \\
II & $11(52.4)$ \\
III & $8(38.1)$ \\
No. of tumors $(\%)$ & \\
1 & $12(57.1)$ \\
$>1$ & $9(42.9)$ \\
Recurrence status, $\mathrm{n}(\%)$ & \\
Primary & $12(57.1)$ \\
Recurrent & $9(42.9)$ \\
CIS presence, $\mathrm{n}(\%)$ & \\
None & $16(76.2)$ \\
Presence & $5(23.8)$ \\
Recurrent after TUR, $\mathrm{n}(\%)$ & \\
None & $11(52.4)$ \\
Recurrence & \\
Treatment after TUR, $\mathrm{n}(\%)$ & \\
None & \\
BCG & \\
2nd TUR & \\
Total cystectomy & \\
\hline
\end{tabular}

TUR, transurethral resection; BCG, bacillus calmette-guérin; CIS, carcinoma in situ; T stage, tumor stage.

was stained within the tumor and the stroma at x100 magnification (Fig. 1B).

Statistical analysis. All statistical analyses were performed using StatView (ver. 5.0; SAS Institute Inc., Cary, NC, USA). Mann-Whitney U tests and Student's t-tests were used to evaluate the correlations among TAM, MVCs and clinicopathological features, including age, recurrence status, carcinoma in situ (CIS) presence, recurrent after TUR and treatment after TUR. The correlations among angiogenic factors, MVCs and TAMs were determined by Spearman's rank correlation tests. $\mathrm{P}<0.05$ was considered to indicate a statistically significant difference.

\section{Results}

Patterns of macrophage infiltration and $M V C$. The present study first evaluated the localization of macrophages within the tumor specimens. The patterns of macrophage infiltration and MVCs according to TAM and CD34 counts and ratios 
Table II. Pattern of macrophage infiltration and MVCs according to TAM (CD68 and CD163) and CD34 counts and ratios.

\begin{tabular}{|c|c|c|c|}
\hline Factor & $\begin{array}{l}\text { Count/ratio in the tumor, } \\
\text { mean (range) }\end{array}$ & $\begin{array}{c}\text { Count/ratio in the stroma, } \\
\text { mean (range) }\end{array}$ & $\begin{array}{l}\text { Total count/ratio, } \\
\text { mean (range) }\end{array}$ \\
\hline CD68 & $83.6(3-272)$ & $110.0(23-482)$ & $193.6(53-547)$ \\
\hline CD163 & $48.1(1-167)$ & $85.0(3-407)$ & $133.1(28-445)$ \\
\hline $\mathrm{CD}_{163} / 68^{+}$ & $0.49(0.10-0.89)$ & $0.72(0.37-1.00)$ & $0.66(0.25-0.95)$ \\
\hline CD34 & & & $36.0(8-87)$ \\
\hline $\mathrm{CD} 68^{+} / 34^{+}$ & & & $7.13(1.01-20.00)$ \\
\hline $\mathrm{CD} 163^{+} / 34^{+}$ & & & $4.57(0.93-13.13)$ \\
\hline
\end{tabular}

TAM, tumor-activated macrophage; MVC, microvessel count; CD, cluster of differentiation.
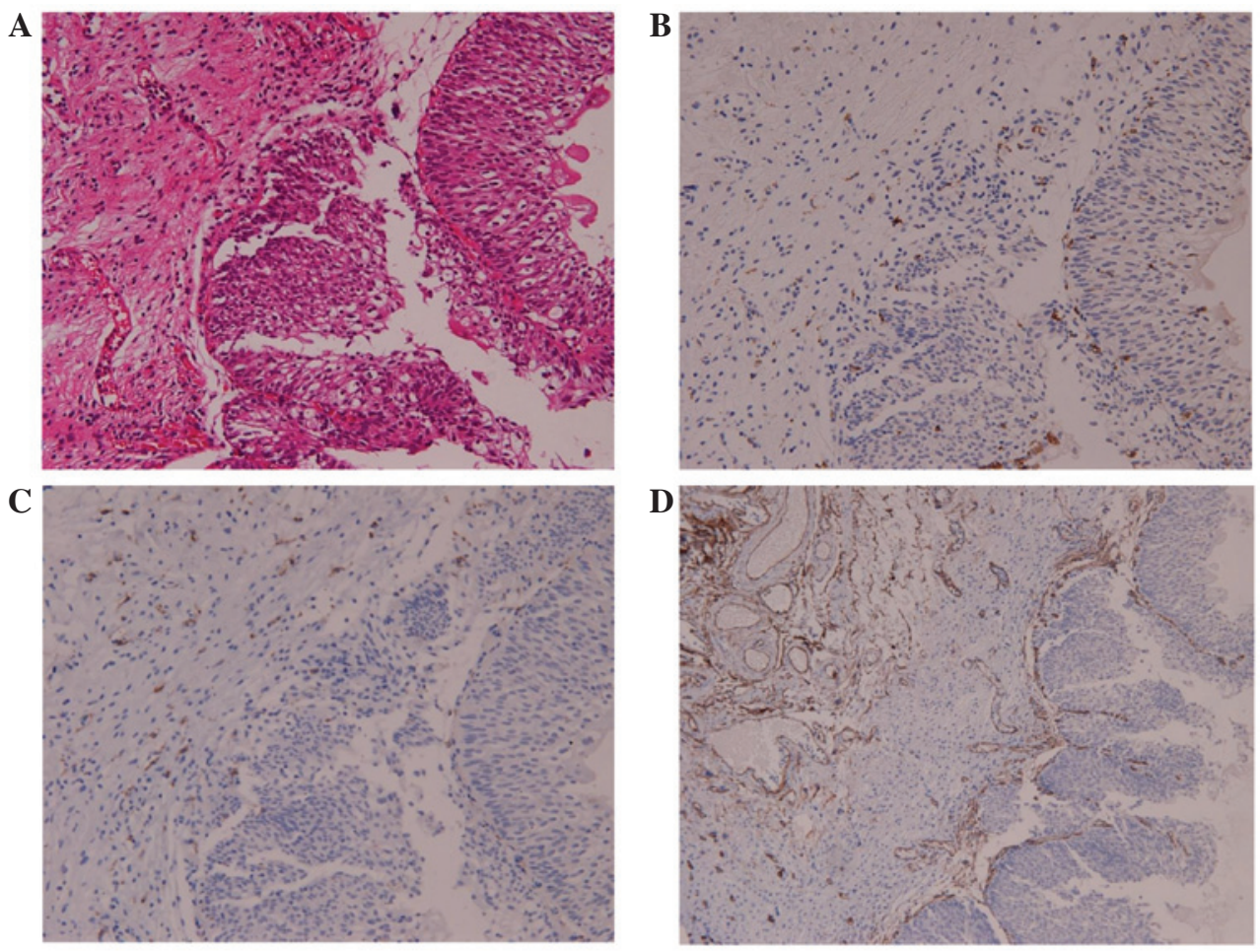

Figure 1. Immunohistochemical staining for TAMs and MVCs in bladder cancers. Representative distribution patterns of TAMs and MVCs in bladder cancers are shown. (A) Hematoxylin and eosin staining was used to perform a histomorphological evaluation, while TAM and M2 macrophages were stained using (B) anti-CD68 and (C) anti-CD163 monoclonal antibodies in the bladder cancer specimens (original magnification, x200). (D) Microvessels stained for invasive bladder cancer using anti-CD34 monoclonal antibodies (original magnification, x100). TAM, tumor-activated macrophage; MVC, microvessel count; $\mathrm{CD}$, cluster of differentiation.

are shown in Table II. CD68 ${ }^{+}$and $\mathrm{CD} 163^{+}$macrophages were present in both the tumor stroma and tumor islets. The tumor stroma included the papillary axis, lymphoid aggregates and the stroma. The mean count of $\mathrm{CD}^{+} 8^{+}$macrophages was 110.0 in the stroma, 83.6 in the tumor and 193.7 in total, whereas the mean number of $\mathrm{CD} 163^{+}$macrophages was 85.0 in the stroma, 48.1 in the tumor and 133.1 in total. The mean ratio of $\mathrm{CD} 163^{+} / \mathrm{CD} 68^{+}$macrophages was 0.49 in the tumor, 0.72 in the associated stroma and 0.66 in total. Next, the microvessel density (MVD) within the tumor specimens was examined by staining with anti-CD34 antibodies. CD34 was present in the total tumor and its associated stroma; the mean CD34 count was 36 . The mean $\mathrm{CD} 68^{+} / \mathrm{CD} 34^{+}$and $\mathrm{CD} 163^{+} / \mathrm{CD} 34^{+}$ratios in the tumor were 7.13 and 4.57 .
Correlation among clinicopathological characteristics and TAMs and MVCs in bladder cancer. Next, the correlation among the clinicopathological characteristics and the $\mathrm{CD}^{+} 8^{+}$and $\mathrm{CD} 163^{+}$macrophages, and $\mathrm{CD} 34^{+}$microvessels, was assessed. The correlations between clinical variables and macrophage counts are presented in Fig. 2. The mean $\mathrm{CD} 63^{+} / \mathrm{CD} 68^{+}$ratio in the stage $\mathrm{T} 2$ bladder tumors (0.68) was significantly higher than that in the stage Ta tumors (0.39) (Fig. 2Aa; $\mathrm{P}=0.0384$ ). The mean ratio of $\mathrm{CD} 63^{+} / \mathrm{CD} 68^{+}$was significantly higher in the total tumor tissues of the stage T2 bladder cancers (0.71) compared with that in the stage Ta cancers (0.56) (Fig. 2Ab; $\mathrm{P}=0.0474$ ). The mean $\mathrm{CD} 163^{+} / \mathrm{CD} 68^{+}$ratio was significantly higher in the grade III bladder tumors $(0.69)$ than in the grade I $(0.43)$ 
Aa

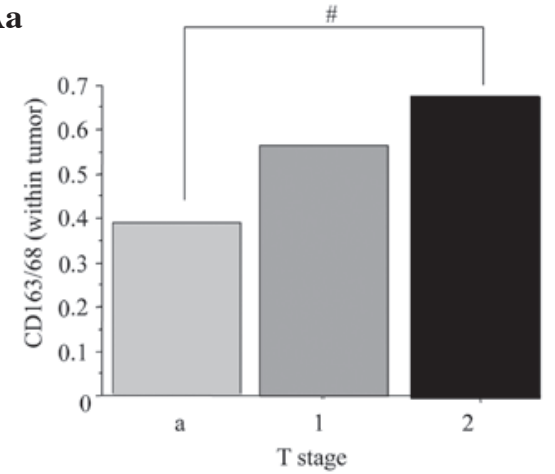

c

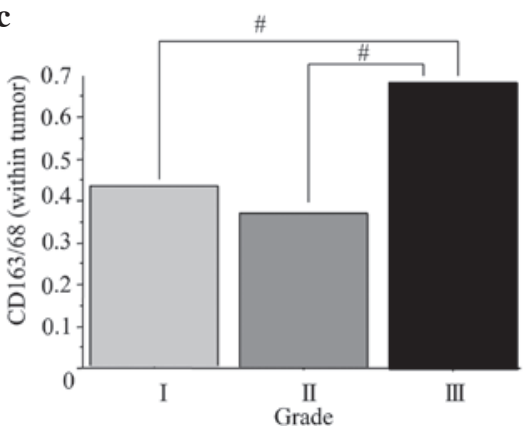

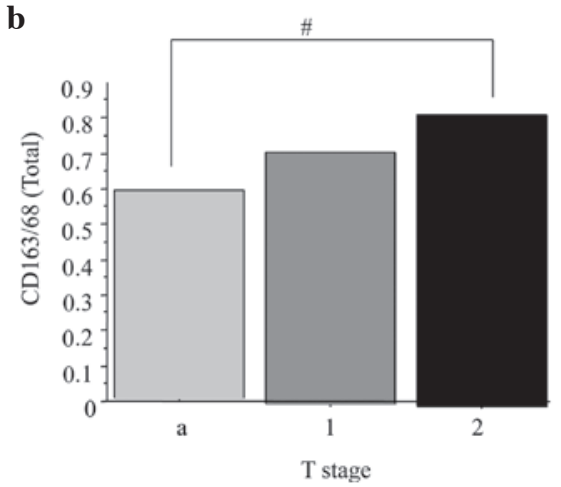

d

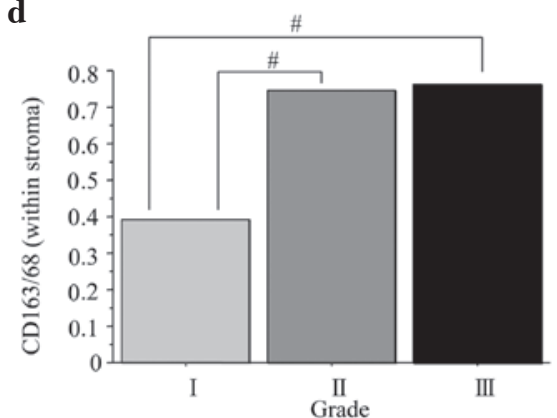

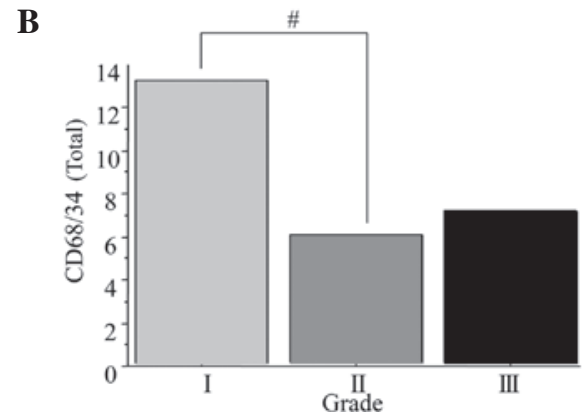

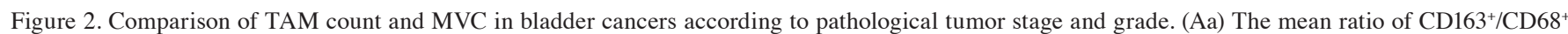

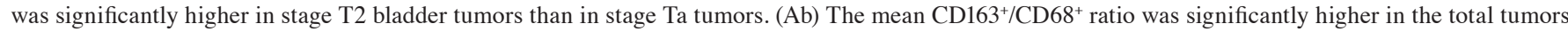
of stage T2 bladder tumors than stage Ta tumors. (Ac) The mean CD163 ${ }^{+} \mathrm{CD} 68^{+}$ratio was significantly higher in grade III bladder tumors compared with grade I and II tumors. (Ad) The mean $\mathrm{CD} 163^{+} / \mathrm{CD} 68^{+}$ratio was significantly lower in the stroma of grade I bladder cancers compared with grade II and III tumors. (B) The mean $\mathrm{CD} 68^{+} / \mathrm{CD} 34^{+}$ratio was significantly higher in the total tumors of grade I bladder cancers compared with grade II. ${ }^{*} \mathrm{P}<0.05$. TAM, tumor-activated macrophage; MVC, microvessel count; $\mathrm{CD}$, cluster of differentiation.

and II tumors (0.37) (Fig. 2Ac; $\mathrm{P}=0.0320$ ). The mean ratio of $\mathrm{CD} 163^{+} / \mathrm{CD} 68^{+}$within the tumor stroma of grade I (0.39) bladder cancers was significantly lower compared with that in the grade II (0.75) and III (0.76) tumors (Fig. 2Ad; P=0.0451). The mean $\mathrm{CD} 68^{+} / \mathrm{CD} 34^{+}$ratio was significantly higher in the total tumors of the grade I bladder cancers (13.2) than in the grade II (5.8) specimens (Fig. 2B; $\mathrm{P}=0.0492$ ). These results suggested that the higher ratios of $\mathrm{CD}_{163} / \mathrm{CD}^{+} 8^{+}$ macrophages within the stroma, tumor and total tissues were correlated with a higher tumor stage and grade. In addition, the low $\mathrm{CD} 68^{+} / \mathrm{CD} 34^{+}$microvessel ratio was correlated with a higher tumor stage. Conversely, no correlations were found between age, multifocality, recurrence status, CIS presence, recurrence after TUR and treatment after TUR (data not shown).

Correlation between TAM counts and MVCs in bladder cancer. Finally, the correlation between TAM counts and MVCs was examined using Spearman's correlation test.

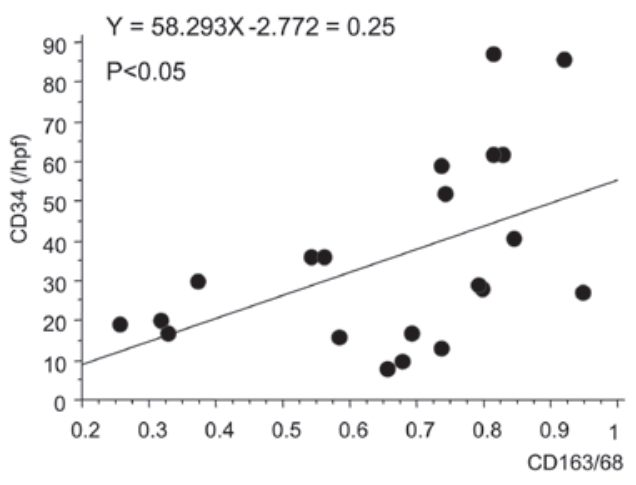

Figure 3. Correlation between tumor-associated macrophage (CD163/68) and microvessel (CD34) counts. CD, cluster of differentiation; hpf, high-power field.

There was a positive correlation $\left(r^{2}=0.25 ; \mathrm{P}=0.0209\right)$ between TAM counts $\left(\mathrm{CD} 163^{+} / \mathrm{CD}^{2} 8^{+}\right.$ratio) and MVCs $(\mathrm{CD} 34$ count $)$ in bladder cancer, suggesting that TAM may be involved in tumor angiogenesis in bladder cancers (Fig. 3; $\mathrm{P}=0.0209$ ). 


\section{Discussion}

The present study examined the correlations between TAM and the predominance of M2-polarized macrophages in bladder cancer, angiogenesis and clinicopathological features. It was demonstrated that the density of M2-polarized macrophages was significantly higher in invasive bladder cancers than in non-muscle invasive cancers. In addition, the MVC distribution was higher in higher grade bladder cancers, and the predominance of M2-polarized macrophages correlated positively with MVCs in bladder cancer.

TAMs are a major component of tumor immune infiltrates (18). A previous study reported that there were more TAMs in invasive bladder cancers than in non-muscle invasive cancers (19), and that there was a positive correlation between TAMs and MVD. These findings suggest that TAMs with an angiogenic or infiltrating phenotype are differentially expressed in invasive or non-muscle invasive bladder cancers. However, the process by which TAMs become angiogenic or infiltrating is unclear. Hypoxia, a common process in tumor tissues, may be one of the mechanisms that is involved in the activation of TAMs. The migration of angiogenic factor-secreting TAMs may be partially aided by reduced oxygen tension in tumor tissues, which subsequently promotes tumor angiogenesis and invasion. It is also possible that invasive bladder cancers stimulate the deep migration of TAMs into tumors and the secretion of a number of angiogenic factors.

However, previous findings have been based on the analysis of CD68. TAMs assume an M2-like phenotype, which is associated with tumor promotion $(18,20)$. Extensive TAM infiltration is typically associated with angiogenesis and a poor prognosis in a variety of human cancers (21-24). However, macrophages polarization results in two distinct functional forms: M1 and M2 $(5,6,25)$. Type 1 helper cells (Th1) are activated by classical or M1 macrophages, and exhibit the ability to kill pathogens and create interleukin (IL)-2, IL-12 and pro-inflammatory cytokines for the promotion of responses such as cytotoxic T-cell activation (25). By contrast, low levels of IL-12 and high levels of IL-4 and IL-10 are expressed by alternatively-activated M2 macrophages, promoting Th2 cytokines and inhibiting Th1 responses (7). However, multiple subtypes of M2-polarized macrophages are associated with tumors, which may contribute to immunosuppression, angiogenesis, cell invasion and metastasis depending on the microenvironment $(5,26)$. The identification of CD163 ${ }^{+}$ macrophages has also been shown to be associated with a poor prognosis in several types of cancer $(27,28)$. However, the current study is the first to suggest that the M2 subtype may be a characteristic of bladder tumors that are invasive, with a high tumor grade and microvessels.

The current study also revealed a positive correlation between TAM count and MVCs. This finding suggests that TAMs have direct relevance to tumor angiogenesis through the secretion of angiogenic factors in bladder cancer, although other angiogenic factors from the tumor and stromal components may exhibit a primary role in angiogenesis. It was previously reported that tumor angiogenesis, determined according to MVD, was a significant and independent prognostic indicator in patients with breast and prostate cancer $(29,30)$. In addition, tumor angiogenesis (as determined using MVCs) was an independent prognostic indicator in patients with invasive bladder cancer (31). These findings support the current conclusions, whereby TAM may be of value as a prognostic factor in bladder cancer.

The limitations of the present study include the small sample size and the fact that the study was retrospective and observational, using only existing materials. Therefore the present study did contain a comparison between healthy and cancerous tissues. Accordingly, an interventional study should be performed, using normal bladder tissue from patients as control samples, in order to confirm the present findings.

The current study demonstrated an association between the polarized M2 TAM phenotype and MVCs, and the pathological outcome, tumor grade and invasiveness. Therefore, more aggressive therapeutic strategies than TUR may be recommended in patients who have a high M2 count and MVC. However, prior to clinical use of this strategy, further studies with large population sizes and healthy control groups are required in order to determine the optimal treatment for such patients.

\section{Acknowledgements}

The authors would like to thank Enago (www.enago.jp) for reviewing the English language of the original manuscript.

\section{References}

1. DeSantis CE, Lin CC, Mariotto AB, Siegel RL, Stein KD, Kramer JL, Alteri R, Robbins AS and Jemal A: Cancer treatment and survivorship statistics, 2014. CA Cancer J Clin 64: 252-271, 2014.

2. van Rhijn BW, Burger M, Lotan Y, Solsona E, Stief CG, Sylvester RJ, Witjes JA and Zlotta AR: Recurrence and progression of disease in non-muscle-invasive bladder cancer: From epidemiology to treatment strategy. Eur Urol 56: 430-442, 2009.

3. Babjuk M, Oosterlinck W, Sylvester R, Kaasinen E, Böhle A, Palou-Redorta J and Rouprêt M; European Association of Urology (EAU): EAU guidelines on non-muscle-invasive urothelial carcinoma of the bladder, the 2011 update. Eur Urol 59: 997-1008, 2011.

4. Qian BZ and Pollard JW: Macrophage diversity enhance stumor progression and metastasis. Cell 141: 39-51, 2010.

5. Sica A, Larghi P, Mancino A, Rubino L, Porta C, Totaro MG, Rimoldi M, Biswas SK, Allavena P and Mantovani A: Macrophage polarization in tumor progression. Semin Cancer Biol 18: 349-355, 2008.

6. Mantovani A, Sozzani S, Locati M, Allavena P and Sica A: Macrophage polarization: Tumor-associated macrophages as a paradigm for polarized M2 mononuclear phagocytes. Trends Immunol 23: 549-555, 2002.

7. Folkman J and Klagsbrun M: Angiogenic factor. Science 235: 442-446, 1987.

8. Folkman J and Shing Y: Angiogenesis. J Biol Chem 267: 10931-10934, 1992.

9. Sunderkötter C, Steinbrink K, Goebeler M, Bhardwaj R and Sorg C: Macrophages and angiogenesis. J Leukoc Biol 55: 410-422, 1994.

10. Leek RD, Harris AL and Lewis CE: Cytokine network in solid human tumors regulation of angiogenesis. J Leukoc Biol 56: 423-435, 1994.

11. Mosser DM and Edwards JP: Exploring the full spectrum of macrophage activation. Nat Rev Immunol 8: 958-969, 2008.

12. Murray PJ and Wynn TA: Obstacles and opportunities for understanding macrophage polarization. J Leukoc Biol 89: 557-563, 2011.

13. Allavena P, Sica A, Solinas G, Porta C and Mantovani A: The inflammatory micro-environment in tumor progression: The role of tumor-associated macrophages. Crit Rev Oncol Hematol 66: $1-9,2008$ 
14. Hao NB, Lu MH, Fan YH, Cao YL, Zhang ZR and Yang SM: Macrophages in tumor microenvironments and the progression of tumors. Clin Dev Immunol 2012: 948098, 2012.

15. Tang X: Tumor-associated macrophages as potential diagnostic and prognostic biomarkers in breast cancer. Cancer Lett 332: 3-10, 2013.

16. Shabo I and Svanvik J: Expression of macrophage antigens by tumor cells. Adv Exp Med Biol 714: 141-150, 2011

17. Sobin LH and Wittekind C (eds): TNM Classification of Malignant Tumours (UICC). 6th edition. Wiley-Blackwell, Hoboken, NY, 2002.

18. Mantovani A and Sica A: Macrophages, innate immunity and cancer: Balance, tolerance and diversity. Curr Opin Immunol 22 231-237, 2010.

19. Hanada T, Nakagawa M, Emoto A, Nomura T, Nasu N and Nomura Y: Prognostic value of tumor-associated macrophage count in human bladder cancer. Int J Urol 7: 263-269, 2000.

20. Siveen KS and Kuttan G: Role of macrophages in tumor progression. Immunol Lett 123: 97-102, 2009.

21. Talmadge JE, Donkor M and Scholar E: Inflammatory cell infiltration of tumors: Jekyll or Hyde. Cancer Metastasis Rev 26: 373-400, 2007

22. Koide N, Nishio A, Sato T, Sugiyama A and Miyagawa S: Significance of macrophage chemoattractant protein-1 expression and macrophage infiltration in squamous cell carcinoma of the esophagus. Am J Gastroenterol 99: 1667-1674, 2004.

23. Lissbrant IF, Stattin P, Wikstrom P, Damber JE, Egevad L and Bergh A: Tumor associated macrophages in human prostate cancer: Relation to clinicopathological variables and survival. Int J Oncol 17: 445-451, 2000.
24. Ohno S, Ohno Y, Suzuki N, Kamei T, Koike K, Inagawa H, Kohchi C, Soma G and Inoue M: Correlation of histological localization of tumor-associated macrophages with clinicopathological features in endometrial cancer. Anticancer Res 24: 3335-3342, 2004

25. Mantovani A and Locati M: Tumor-associated macrophages as a paradigm of macrophage plasticity, diversity and polarization: Lessons and open questions. Arterioscler Thromb Vasc Biol 33: 1478-1483, 2013.

26. Shimura S, Yang G, Ebara S, Wheeler TM, Frolov A and Thompson TC: Reduced infiltration of tumor-associated macrophages in human prostate cancer: Association with cancer progression. Cancer Res 60: 5857-5861, 2000.

27. Medrek C, Pontén F, Jirström K and Leandersson K: The presence of tumor associated macrophages in tumor stroma as a prognostic marker for breast cancer patients. BMC Cancer 12: 306, 2012.

28. Zhang QW, Liu L, Gong CY, Shi HS, Zeng YH, Wang XZ, Zhao YW and Wei YQ: Prognostic significance of tumor-associated macrophages in solid tumor: A meta-analysis of the literature. PLoS One 7: e50946, 2012.

29. Weidner N, Semple JP, Welch WR and Folkman J: Tumor angiogenesis and metastasis-correlation in invasive breast carcinoma. N Engl J Med 324: 1-8, 1991.

30. Weidner N, Carroll PR, Flax J, Blumenfeld W and Folkman J: Tumor angiogenesis correlates with metastasis in invasive prostatic carcinoma. Am J Pathol 143: 401-409, 1993.

31. Bochner BH, Cote RJ, Weidner N, Groshen S, Chen SC, Skinner DG and Nichols PW: Angiogenesis in bladder cancer: Relationship between microvessel density and tumor prognosis. J Natl Cancer Inst 87: 1603-1612, 1995. 